\title{
A Combined Task Analysis Method for Data Selection in Mandarin Isolated Word Recognition System
}

\author{
Z. Y. He, Z. G. Wang, W. Li, and J. Wu \\ Department of Electronic Engineering, Tsinghua University, Beijing \\ Iflytek Speech Lab at the Department of EEIS at USTC, Hefei \\ zyhe_ts@mail.tsinghua.edu.cn, zgwang@iflytek.com \\ liwei1976@gmail.com,wuji_ee@tsinghua.edu.cn
}

\begin{abstract}
This paper studies the performance of the data selection with a combined task analysis method in task adaptation on Mandarin isolated word recognition. The proposed task analysis method combines coverage unit balanced task analysis with the confusability based analysis. The performance is evaluated with several experiments.
\end{abstract}

Index Terms - task analysis, data selection, adaptation, isolated word recognition

\section{Introduction}

Task adaptation in speech recognition technology is generally necessary to collect a set of task specific (indomain) adaptive data. In many realistic application scenarios, a large amount of speech data can be pooled together as the candidate data for the adaptive data selection. One of choices is to use all data as the adaptive data. However it is not necessary to achieve the best performance improvement due to the mismatch of the acoustic unit (coverage unit) distributions between the task and the candidate data. Moreover in many realistic word isolate ASR applications, the task information can be obtained such as the task vocabulary, the occurrence frequency of different words in the task vocabulary etc. So it makes sense to select the adaptive data based on task analysis on such information of the coverage unit.

In [1], adaptation script is generated with two stages including 1) coverage unit distribution derived by task analysis; 2) script generation based on task dependent coverage unit distribution. In [2], all context-independent (CI) INITIAL/FINAL in the task are used as the coverage units and the distribution of the coverage unit occurrence in the task then obtained. The adaptation script is generated by covering all coverage units with minimum number of the sentences firstly and approximating the distribution of the coverage units by the adaptation script secondly. The distribution dissimilarity measure is defined as the normalized inner product. In [3], phoneme is used as the coverage unit and KULLBACK-LEIBLER (KL) divergence as the distribution measure instead. In [1], triphone and context dependent state are used as the coverage units by considering more context information during the adaptation script generation. An active approach based on automatic analysis of task vocabulary is used to generate the coverage unit distribution in which the confusability existing in the task is analyzed and used in the generation of adaptation script.

Similar procedure as above can also be used in the adaptive data selection with only difference by replacing the stage of the script generation with the data selection based on the result of the task analysis. In this paper, the coverage unit balanced task analysis method is combined with the confusability analysis based method for the adaptation data selection and the corresponding performances are compared with both coverage unit balanced approach and confusability analysis based method. In addition, there is also another important issue about which type of coverage unit should be used. In this paper, three different types of coverage units namely right context dependent Initial/toned Final (RCI/TF), syllable and word are compared in the data selection for the task adaptation on the Mandarin isolated word recognition.

In the next section, the procedure of data selection with the combined task analysis method is presented. In section 3 , such data selection procedure is evaluated with the corresponding experiments. In the last section, the conclusions and corresponding discussions are given.

\section{Data Selection with Automatic Task} analysis by Combining Coverage unit 


\section{balanced approach with confusability analysis based approach}

Let $V=\left\{v_{i} \mid i=1,2, \cdots, N_{v}\right\}$ denote the set of words in the task vocabulary and $P_{t}^{o c c}\left(v_{i}\right)$ represent the occurrence frequency of $v_{i}$. Let further $V_{c}$ denote the subset of the confusable words in $V$ and $U=\left\{u_{k} \mid k=1,2, \cdots, N_{u}\right\} \quad$ denote the set of coverage units. Different kinds of coverage units are adopted namely word, syllable or $R C I / T F$ respectively so as to compare the influence of different levels of context information on the task analysis. The data selection procedure by combining two task analysis methods, namely coverage unit balanced task analysis and task confusability based analysis is presented as follows:

Step 1: Coverage Unit Balanced Task Analysis (B_TA)

\section{Initialization}

Initialize $V_{c}$ as an empty set.

2. Calculation of Task Coverage Unit Occurrence Probability Distribution

Calculate the task coverage unit occurrence probability distribution as follows:

$P_{t}\left(u_{k}\right)=\frac{\sum_{j} P_{t}^{o c c}\left(v_{j}\right) \operatorname{count}_{j}\left(u_{k}\right)}{\sum_{j, l} P_{t}^{o c c}\left(v_{j}\right) \operatorname{count}_{j}\left(u_{l}\right)}$

where cout $_{j}\left(u_{k}\right)$ is the count of $u_{k}$ in $v_{j}$.

\section{Step 2: Confusability Task Analysis (C_TA)}

\section{Initialization}

Initialize $V_{c}$ as an empty set.

\section{Identification of Confusable Words}

For each pair of vocabulary words $\left(v_{i}, v_{j}\right)$, if

$\operatorname{SIM}\left(v_{i}, v_{j}\right)<T H_{c}$, do

- Update $V_{c}=V_{c} \cup\left\{v_{i}, v_{j}\right\}$

- For $v_{i}$ and $v_{j}$, do

$$
C\left(v_{k}\right)=C\left(v_{k}\right)+1, k=i, j .
$$

where
$\operatorname{SIM}\left(v_{i}, v_{j}\right)=\frac{1}{2}\left[D_{H M M}\left(\lambda_{i}, \lambda_{j}\right)+D_{H M M}\left(\lambda_{j}, \lambda_{i}\right)\right]$ and $D_{H M M}\left(\lambda_{i}, \lambda_{j}\right)$ is an asymmetric dissimilarity measure between $\lambda_{i}$ and $\lambda_{j}$ as defined in [6] in which a specific pronunciation of a word is represented as a sequence of coverage unit, and modeled by an LR-CDHMM $\lambda_{i}$.

3. Calculation of Confusable Distribution

Calculate the confusable probability distribution of words as follows:

$$
P_{t}^{c n f}\left(v_{k}\right)=\frac{C\left(v_{k}\right)}{\sum_{l} C\left(v_{l}\right)}, v_{k} \in V_{c}
$$

Step 3: Combine B_TA with $C_{-} T A$

$P_{t}\left(v_{k}\right)=\left\{\begin{array}{l}\lambda \cdot P_{t}^{c n f}\left(v_{k}\right)+(1-\lambda) \cdot P_{t}^{o c c}\left(v_{k}\right), v_{k} \in V_{c} \\ (1-\lambda) \cdot P_{t}^{o c c}\left(v_{k}\right), v_{k} \notin V_{c}\end{array}\right.$ where $\lambda \in[0,1]$. We can observe that the target probability distribution is the same as the results of B_TA when $\lambda=0$. If $\lambda=1$, only the confusable probability distribution will be used.

Step 4: Data Selection

Given $P_{t}\left(v_{k}\right) \quad v_{k} \in V_{c}$, a set of task dependent adaptation data is selected from a pool of candidate data set according to the KullbackLeibler measure with detail description presented in [3].

\section{Experiments and Results}

\subsection{Experimental Setup}

In order to evaluate the performance of the proposed data selection method, a Mandarin isolated word recognition application is used as our task. The basic speech units of our ASR system are RCI/TF which consists of 253 basic units. Each right-context-initial basic unit is modeled by a three-emitting-state LRCDHMM without state skipping and the toned-final unit is modeled by a five-emitting-state LR-CDHMM without state skipping. Each state has 12 Gaussians mixture components with each component having a diagonal covariance matrix. A special three-state CDHMM is also used for silence modeling. The 43- 
dimensional feature vector used in this study consists of 12-dimension MFCC and log-scaled energy normalized by the average energy of the individual sentence, plus their first and second order derivatives. Sentence-based cepstral mean subtraction is applied for acoustic normalization both in training and testing. Moreover there are also 4-dimensional tone features [7]. HTK toolkit [5] is used to train a set of speakerindependent (SI) RCI/TF with 1065 states in total. The training data consists of about 142-hour speech data including 1,106k Chinese syllables.

\subsection{Experimental Evaluation}

The total adaptation and testing data consists of 6.57 hours speech data consisting of 2079 Chinese words. In order to accurately evaluate the different methods, 10 -fold cross-validation is adopted with the best performance shown in [4]. Let $D=\left\{x_{1}, x_{2}, \cdots x_{n}\right\}$ be a dataset with $n$ labeled instances. In 10-fold cross validation, $D$ is randomly split into 10 mutually exclusive subsets (the folds) $D_{1}, D_{2}, \cdots, D_{10}$. There are 10 experiments during the evaluation. In the $i^{t h}$ experiment, the subset $D_{i}$ is used as the testing set and the others are used as adaptation set which is represented by $D / D_{i}$. Let $y_{i j}$ represents the label of the $j^{\text {th }}$ instance $x_{i j}$ in the subset $D_{i}$. Then the crossvalidation estimation of accuracy is

$$
a c c=\frac{1}{n} \sum_{\langle i, j\rangle} \delta\left(I\left(D / D_{i}, t_{i j}\right), y_{i j}\right)
$$

where $I\left(D / D_{i}, t_{i j}\right)$ denotes the recognized label for the $j^{\text {th }}$ instance $x_{i j}$ in the subset $D_{i}$, using the model which is adapted with the adaptation data set $D / D_{i}$ from the original (task independent) model. $\delta(\bullet)$ is Kronecker delta function.

\subsection{Adaptation Experiments and Results}

Given the above set of task independent CDHMMs and for each testing set $D_{i}$, several adaptation data set with different number of utterances can be generated to achieve a similar distribution of coverage units to the target distribution. Moreover a fixed threshold
$T H_{c}=5$ is used for the confusability analysis in the data selection in all experiments.

Given the task independent CDHMM systems, word accuracy $80.33 \%$ can be obtained and can be improved as $87.99 \%$ by using all candidate data as the adaptation data. Starting from the task independent CDHMM systems, a series of supervised batch-mode task adaptation experiments are conducted for each testing set by using two-pass adaptation strategies. In the first pass, a standard MLLR adaptation algorithm with global transformation is done and the transformed model is derived as the seed model in the second pass. In the second pass, the MLLR adaptation algorithm with multiple regression classes are again used which is entailed together with MAP adaptation. In all experiments, a common regression tree with 149 leaf nodes derived from task independent CDHMM is used and the block diagonal transformation matrices are used and the threshold of the occupation counts is set to be 700.0 . The scaling factor for MAP adaptation is set to be 12.0 .

In order to compare the effectiveness of the different coverage units in the data selection, the first experiment is used which is shown in Fig. 1. Three coverage units, namely word, syllable and $R C I / T F$ are used by only using $\boldsymbol{B}_{-} \boldsymbol{T} \boldsymbol{A}$ as the task analysis strategy. It is observed in Fig. 1 that using both word and syllable as coverage unit require much less data to achieve the same performance by using all data. For example, using syllable as coverage unit archives a word recognition accuracy of $88 \%$ with about 2000 utterances as adaptation data. Using word as coverage unit performs better with only about 1000 utterances. Moreover, word performs better than other two coverage units and $\mathrm{RCI} / \mathrm{TF}$ performs worst. It means more context information is beneficial for the adaptation data selection. It is also observed that more adaptation data is still helpful for the improvement of the recognition performance for the syllable and $\mathrm{RCI} / \mathrm{TF}$ as the coverage unit. But such improvement declines quickly or even no improvement exists with increasing adaptation data for the word as the coverage unit. The reason is that the amount of the candidate data is limited in which only part of data is beneficial to decrease the dissimilarity between the target distribution probability and that of selected adaptation data. As a result, no improvement can be obtained on the word recognition accuracy. 


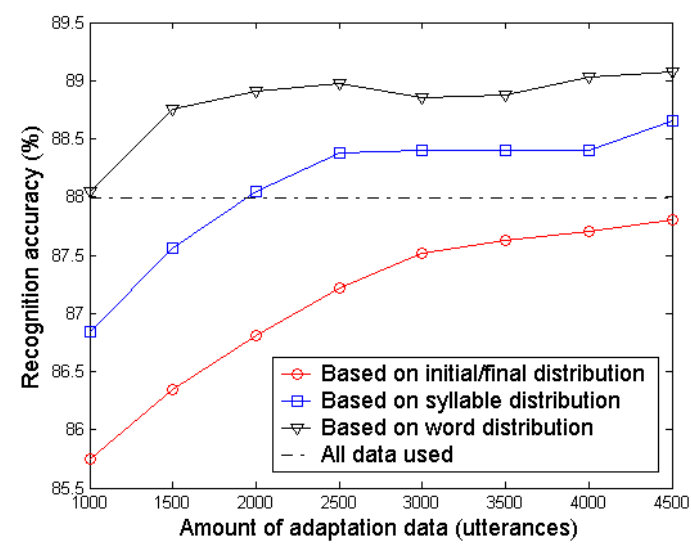

Fig. 1. Adaptation performance using data selection based on Coverage Unit Balanced Task Analysis (use 3 types of coverage unit distribution). The acc. of baseline is $87.99 \%$.

Since the word performs best among the three coverage units, the second experiment only uses the word as the coverage unit to evaluate the data selection with the combined task analysis approach. Table 1 demonstrates the performances with the different values set by $\lambda$ for data selection in which all the experiments use word as coverage unit due to its best performance demonstrated in Fig. 1. It is observed that there is no significant performance difference between B_TA $(\lambda=0)$ and $C_{-}$TA when $\lambda$ take the value of $0.2,0.5,0.8$. When the number of utterances of adaptation data exceeds 2500 , C_TA perform a slightly better than B_TA. Moreover only using C_TA $(\lambda=1.0)$ as the task analysis for the data selection performs worse than other task analysis methods. Such behavior is quite different from [1] which is due to the different natures of the testing data sets. The confusable/non-confusable utterances in the testing data sets used in this paper is much more indistinguishable than that used in [1]. So the current confusability analysis strategy is not good enough for such more complex task.

\section{Conclusion}

This paper studies the data selection method by combing two task analysis strategies namely coverage unit balanced task analysis and confusability analysis based task analysis. The experiment shows that the combined task analysis performs a slightly better than only using coverage unit balanced task analysis. But such improvement is not significant. Moreover the confusability based task analysis performs worst mainly due to more complexity in the task comparing with [1], which means further study is necessary on the confusability based task analysis. In addition, three different coverage units namely RCI/TF, syllable and word, are compared and the word performs best among them which means the data selection can benefit a lot from more context information.

Table. 1. Adaptation performance using data selection based on Confusion Task Analysis. The acc. of baseline is $87.99 \%$. All the experiments are based on word distribution. Notice that the results (when $\lambda=0$ ) is just that of B_TA using word as the coverage units displayed in Fig.1.

\begin{tabular}{|l|c|c|c|c|c|}
\hline \multirow{2}{*}{} & \multicolumn{5}{|c|}{ Acc.\% (different utterances of adapt. data) } \\
\cline { 2 - 6 } & 1000 & 1500 & 2000 & 2500 & 3000 \\
\hline$\lambda=0$ & 88.05 & 88.75 & 88.91 & 88.97 & 88.85 \\
\hline$\lambda=0.2$ & 87.99 & 88.77 & 88.76 & 89.07 & 88.91 \\
\hline$\lambda=0.5$ & 88.04 & 88.55 & 88.73 & 89.03 & 88.98 \\
\hline$\lambda=0.8$ & 87.86 & 88.58 & 88.68 & 88.89 & 88.89 \\
\hline$\lambda=1.0$ & 86.30 & 86.39 & 86.64 & 86.68 & 86.66 \\
\hline
\end{tabular}

\section{References}

[1] Q. Huo and W. Li, “An active approach to speaker and task adaptation based on automatic analysis of vocabulary confusability", Proc. INTERSPEECH, 2007, pp. 1569-1572.

[2] Jia lin Shen, Hsin min Wang, Ren yuan Lyu, and Lin shan Lee, "Automatic selection of phonetically distributed sentence sets for speaker adaptation with application to large vocabulary mandarin speech recognition", Computer Speech and Language, Vol. 13, 1999, pp. 79-97.

[3] X. Cui and A. Alwan, "Efficient adaptation text design based on the Kullback-Leibler measure", Proc. ICASSP, 2002, pp. I-613-616.

[4] K. Ron, "A study of cross-validation and bootstrap for accuracy estimation and model selection," Proc. IJCAI, 1995, pp. 1137-1143

[5] S. T. Young, et al., The HTK Book (revised for HTK Version 3.4), 2006.

[6] Q. Huo and W. Li, “A DTW-based dissimilarity measure for left-to-right hidden Markov models and its application to word confusability analysis," Proc. INTERSPEECH, 2006, pp. 2338-2341,

[7] Ch. L. Qiao, Ji Wu, Z. Y. Wang, "The research on applying tone information in Mandarin speech recognition", Computer Engineering and Applications, Vol. 38, 2002, pp. 51-53. 